九州大学学術情報リポジトリ

Kyushu University Institutional Repository

\title{
Mini Review on Recent Applications of Nanotechnology in Nutrient and Heavy Metals Removal from Contaminated Water
}

\section{Falyouna, Omar}

Water and Environmental Engineering Laboratory, Interdisciplinary Graduate School of Engineering Sciences, Kyushu University

Maamoun, Ibrahim

Water and Environmental Engineering Laboratory, Interdisciplinary Graduate School of Engineering Sciences, Kyushu University

Bensaida, Khaoula

Water and Environmental Engineering Laboratory, Interdisciplinary Graduate School of Engineering Sciences, Kyushu University

Mohd Faizul Idham

Water and Environmental Engineering Laboratory, Interdisciplinary Graduate School of Engineering Sciences, Kyushu University

他

https://doi.org/10.5109/4738583

出版情報: Proceedings of International Exchange and Innovation Conference on Engineering \& Sciences (IEICES). 7，pp.161-169，2021-10-21. 九州大学大学院総合理工学府

バージョン :

権利関係 : 


\title{
Mini Review on Recent Applications of Nanotechnology in Nutrient and Heavy Metals Removal from Contaminated Water
}

\author{
Omar Falyouna $^{1}$, Ibrahim Maamoun ${ }^{1}$, Khaoula Bensaida ${ }^{1}$, Mohd Faizul Idham $^{1}$, Yuji Sugihara ${ }^{2}$ Osama Eljamal $^{{ }^{*}}$ \\ ${ }^{1}$ Water and Environmental Engineering Laboratory, Interdisciplinary Graduate School of Engineering Sciences, Kyushu \\ University, 6-1 Kasuga-Koen Kasuga, Fukuoka 816-8580, Japan. \\ ${ }^{2}$ Environmental Fluid Science Laboratory, Interdisciplinary Graduate School of Engineering Sciences, Kyushu \\ University, 6-1 Kasuga-Koen Kasuga, Fukuoka 816-8580, Japan. \\ ${ }^{*}$ Corresponding author email: osama-eljamal@kyudai.jp
}

\begin{abstract}
Several toxic substances, such as nutrients, heavy metals, radionuclides, and pharmaceuticals, are continuously discharging to the environment, as a result of unaccountable industrial and agricultural activities, and contaminating huge quantities of soil and water. Nanotechnology was employed over the past years to remediate the contaminated waters and clean the environment from these pollutants to protect the life on the planet. Nanoscale zerovalent iron (nZVI) is one of the most applied nanomaterials in the field of water and wastewater treatment and it is extensively utilized to remove a wide range of contaminants from water. Thus, this mini review intends to summarize the latest applications of $n Z V I$ and its composites in eliminating several nutrients and heavy metals, namely nitrate $\left(\mathrm{NO}_{3}^{-}\right)$, phosphorous $(\mathrm{P})$, chromium (Cr) and $\operatorname{arsenic}(A s)$.
\end{abstract}

Keywords: Arsenic (As); Chromium (Cr); Nanoscale zerovalent iron (nZVI); Nitrate $\left(\mathrm{NO}_{3}^{-}\right)$, Phosphorous $(P)$.

\section{INTRODUCTION}

The irresponsible anthropogenic activities and the unwise application of agricultural chemicals (e.g. pesticides, fertilizers, herbicides, and insecticides) resulted in contaminating water and soil with toxic contaminants such as nutrients (i.e. Nitrate and phosphorous), heavy metals (e.g. Chromium, arsenic, nickel, cadmium, copper, mercury, zinc, palladium, etc.), and organic compounds [i.e. polyaromatic hydrocarbons (PAHs), perfluorinated organics (PFOs), etc.) [1,2]. Furthermore, the inadequate treatment of different types of wastewater such as domestic wastewater, industrial wastewater, nuclear wastewater, and hospital wastewater is another source for these contaminants and it can introduce another lethal contaminates, for instance, radioactive elements (i.e. Cesium, uranium, iodine, strontium, etc.), personal care products (PCPs), and pharmaceuticals (e.g. antibiotics) [3,4]. Discharge of these contaminants surely will threat all forms of life on the earth (i.e. Humans, animals, microorganisms, etc.) and negatively affect the surrounding environment, especially the aquatic environments (e.g. rivers, lakes, groundwater, etc.) [5,6]. Therefore, it is essential to develop an effective treatment technology to remove these contaminants from the limited water resources and provide a clean drinking water.

Nanotechnology is a promising approach that is getting the attention of many researchers in field of water and wastewater technologies because it has the potentials to overcome the limitations of traditional treatment technologies and it has the ability to meet the strict water regulations [7,8]. Nanoscale Zero-Valent Iron particles (nZVI) are one of the most promising nanomaterials for environmental remediation. Due to its small size, high surface area and high reducing potentials, nZVI, as a versatile material, is widely applied to remove a wide range of contaminants and pollutants $[9,10]$. Thus, this mini review aims to provide a recap for the state-of-theart applications of nZVI and its composites in removing nitrate, phosphorous, chromium and arsenic from water as representative contaminants for nutrients and heavy metals.

\section{NUTRIENT REMOVAL}

\subsection{Nitrate removal}

Water contamination with nitrate $\left(\mathrm{NO}_{3}{ }^{-}\right)$causes many health and environmental complications. For instance, infant methemoglobinemia (blue baby), cancer, and liver diseases can result from drinking water contaminated with nitrate [11]. Moreover, high levels of nitrate contamination can deteriorate the quality of surface water bodies by promoting eutrophication [12]. Hence, the world health organization (WHO) defined the maximum contamination level of nitrate in drinking water as $50 \mathrm{mg}$ $\mathrm{L}^{-1}\left(\mathrm{NO}_{3}^{-}\right)$, whereases the Japanese authorities reduced this level to $10 \mathrm{mg} \mathrm{L}^{-1}(\mathrm{~N})$ [13].

Table 1 summarizes the latest utilization of iron nanoparticles and its composites in removing nitrate from water. Eljamal et al. attempted to improve the performance of $\mathrm{nZVI}$ in removing nitrate from water by coating nZVI with polyacrylamide (PPA) and the coated nZVI (PPA-nZVI) completely removed $300 \mathrm{mg} \mathrm{L}^{-1}$ of nitrate within $2 \mathrm{~h}$ [14]. In addition, table 1 shows that the nZVI-based composites prepared by Song et al. [15], Zhang et al. [16], Zhang et al. [17], Tang et al. [18], Anbia and Kamel [19], and Babaei et al. [20] succeeded to accomplish full removal of different concentrations of nitrate (20-100 $\left.\mathrm{mg} \mathrm{L}^{-1}\right)$, whilst the nZVI-based materials proposed by Khoshro et al. [21], Maamoun et al. [22], Diao et al. [23], He et al. [24], Mokete et al. [25], Li et al. [26], and Valiyeva et al. [27] exhibited a comparable performance with efficiencies ranges from $91.5 \%$ to $99.8 \%$.

\subsection{Phosphorus removal}

Phosphorous (P) is an essential nutrient for the growth of all organisms such as plants, animals, microbes, algae, etc. [28,29]. However, similar to nitrate, excessive concentrations of phosphorus in water will escalate the phenomena of eutrophication and will negatively affect the quality of water and stress the aquatic life [30,31]. Effluents of wastewater treatment plants, landfill 
leachate, fertilizers, and industrial activities are some of the sources for phosphorus contamination [32,33].

Table 2 sums up the recent articles that used nZVI and its composite to remediate phosphorus contaminated water. Eljamal et al. found that the addition of copper chloride salt $\left(\mathrm{CuCl}_{2}\right)$ stimulated the full elimination of 50 $\mathrm{mg} \mathrm{L}^{-1}$ of phosphorus by nZVI [34]. Moreover, Chen et al. proved that stabilizing nZVI with starch strengthened the performance of nZVI to achieve a full uptake of 100 $\mathrm{mg} \mathrm{\textrm {L } ^ { - 1 }}$ of phosphate $\left(\mathrm{PO}_{4}^{-3}\right)$ within an hour [35]. In addition, Marková et al. illustrated that doping a noble metal such as silver (Ag) on the surface of nZVI improved the reactivity of nZVI and the bimetallic ( $\mathrm{Fe}-$ $\mathrm{Ag}$ ) nanoparticles attained a $100 \%$ removal efficiency of $10 \mathrm{mg} \mathrm{L}^{-1}$ of phosphate $\left(\mathrm{PO}_{4}^{-3}\right)$ [36]. Table 2 also demonstrates that several nZVI-based materials for instance, polyacrylamide coated nanoscale zero-valent iron particles (PPA-nZVI) [14], activated carbon supported nanoscale zero-valent iron particles (AC-nZVI) [37], magnesium hydroxide coated-nanoscale zerovalent iron nanoparticles $\left(\mathrm{Fe}^{0}[\mathrm{Mg} / \mathrm{Fe}]^{1.0}\right)$ [38], encapsulated nanoscale zero-valent iron [39] , nanoscale zero-valent iron/nickel supported on zeolite (Z-Fe/Ni) [40], sepiolitenano zero valent iron composite [41], clay-supported nanoscale zero-valent iron [42], nanoscale zerovalent iron particles supported on spondias purpurea seed waste (S-NaOH-NZVI) [43], and nanoscale zerovalent iron particles (nZVI) derived from reducing natural goethite [44], were reported in the literature to efficiently remove high and low concentrations of phosphorus from water.

\section{HEAVY METALS REMOVAL}

\subsection{Chromium removal}

Chromium (Cr) is a heavy metal which used in a wide range of industries for example, pigments and paints, metal finishing, lumber protection, leather tanning, etc. [45]. The excessive industrial application of chromium and the inadequate treatment of industrial wastewater resulted in contaminating surface and groundwater with chromium [46]. Drinking contaminated water with chromium has lethal health issues such as cancer, disorder of nervous system, destruction of liver and kidney and impairment of male reproductive organs [47]. The WHO set the maximum allowable limit of chromium in drinking water and surface water to $50 \mu \mathrm{g} \mathrm{L}^{-1}$ and 100 $\mu \mathrm{g} \mathrm{L}^{-1}$, respectively [48].

Table 3 epitomizes the most recent applications of nZVI and its composites to detoxify chromium-contaminated water. It can be seen from table 3 that most of the applied modifications on nZVI enhanced the elimination of chromium by nZVI with removal efficiencies exceed $99 \%$. For instance, Cheng et al. reported that $\mathrm{CaCO}_{3-}$ coated nZVI removed $100 \%$ of $10 \mathrm{mg} \mathrm{L}^{-1}$ of hexavalent chromium [Cr(VI)] [49]. However, Xu et al. found that chitosan-stabilized biochar supported S-nZVI (CS@BC/S-nZVI) was proficient in removing high concentrations of $\mathrm{Cr}(\mathrm{VI})$ (99.83\% of $100 \mathrm{mg} \mathrm{L}^{-1}$ of $\mathrm{Cr}(\mathrm{VI}))$ [50]. Also, a similar performance was reported by Zhang et al. where more than $99 \%$ of $100 \mathrm{mg} \mathrm{L}^{-1}$ of $\mathrm{Cr}(\mathrm{VI})$ was eliminated by nanoscale zero valent iron supported by biomass-activated carbon (BC-nZVI) [51].

\subsection{Arsenic removal}

Arsenic (As) is a natural heavy metal that exists in earth crust. Beside its natural presence, mining activities and agricultural practices are the main sources for As contamination in the environment [52]. The high toxicity of arsenic made the WHO to consider it as one of chemicals of major public health concerns [53]. Thus, the WHO strictly set the maximum allowable limit of arsenic in drinking water to $10 \mathrm{ppb}$ [54].

Some of the latest utilization of nZVI and its composites in arsenic removal from water are summarized in table 3. According to table 3, most of the fabricated nZVIcomposites were efficient to remove more than $90 \%$ of different concentrations of arsenic from water. For example, stabilization with starch and carboxymethyl cellulose reinforced $\mathrm{nZVI}$ to achieve a complete removal of $2 \mathrm{mg} \mathrm{L}^{-1}$ of arsenate [As(V)] [55]. Likewise, supporting nZVI with activated carbon succeeded in removing $100 \%$ of $2 \mathrm{mg} \mathrm{L}^{-1}$ of $\mathrm{As}(\mathrm{V})$ [56]. On the other hand, using chitosan fiber as a supporting material for nZVI did not improve the removal of $\mathrm{As}(\mathrm{V})$ as chitosan fiber-supported zero-valent iron nanoparticles only removed $70 \%$ of $0.01 \mathrm{mg} \mathrm{L}^{-1}$ of $\mathrm{As}(\mathrm{V})$ [57].

\section{CONCLUSIONS.}

The objective of this mini review is to sum up the recent published articles on the application of nZVI and its composites in cleaning contaminated water with nitrate $\left(\mathrm{NO}_{3}{ }^{-}\right)$, phosphorus $(\mathrm{P})$, chromium $(\mathrm{Cr})$ and arsenic (As). It can be noticed from this review that several improvement techniques were applied on nZVI to overcome the disadvantages of nZVI and to enhance its reactivity towards the removal of the contaminants of interest. For instance, some of the researchers used supporting materials, such as activated carbon, zeolite, multi-wall carbon nanotubes, etc. While, some scientists coated nZVI particles with polymers or dopped another metal (i.e. Copper, nickel, silver) on the surface of nZVI. The purpose of these methods is to distribute and disperse nZVI particles, reduce their aggregation and improve their performance in wastewater treatment applications. This short review concludes that nitrate, phosphorus, chromium and arsenic were efficiently removed by nZVI and its composite from water with removal efficiencies ranges from $90 \%$ to $100 \%$ under different removal conditions. 
Table 1. Application of nanoscale zerovalent iron particles and its composites in nitrate removal from water.

\begin{tabular}{|c|c|c|c|c|}
\hline No. & $\begin{array}{c}\text { Name of the material or } \\
\text { system }\end{array}$ & Removal conditions & $\begin{array}{c}\text { Removal } \\
\text { Efficiency }(\%)\end{array}$ & Reference \\
\hline 1 & $\begin{array}{l}\text { Polyacrylamide coated nZVI } \\
\text { particles } \underline{(\text { PPA-nZVI) }}\end{array}$ & $\begin{array}{l}\text { Nitrate }\left(\mathrm{NO}_{3}^{-}\right) \text {con. }=300 \mathrm{mg} \mathrm{L}^{-1} \text {, initial } \mathrm{pH} \\
=7, \text { material dosage }=1 \mathrm{~g} \mathrm{~L}^{-1} \text {, temperature }= \\
25^{\circ} \mathrm{C} \text {, and contact time }=120 \mathrm{~min} .\end{array}$ & $100 \%$ & [14] \\
\hline 2 & $\begin{array}{l}\text { nZVI/activated carbon micro- } \\
\text { electrolysis }\end{array}$ & $\begin{array}{l}\text { Nitrate }\left(\mathrm{NO}_{3}^{-}\right) \text {con. }=20 \mathrm{mg} \mathrm{L}^{-1}, \text { initial } \mathrm{pH}= \\
(3-7), \mathrm{nZVI} \text { dosage }=4 \mathrm{~g} / \mathrm{L}, \mathrm{nZVI} / \mathrm{C}=2: 1, \\
\text { temperature }=25^{\circ} \mathrm{C}, \mathrm{DO} \text { concentration }<1 \\
\mathrm{mg} \mathrm{L}^{-1}, \text { and contact time }=\text { N/A. }\end{array}$ & $100 \%$ & {$[15]$} \\
\hline 3 & $\begin{array}{l}\text { nZVI supported on pillared } \\
\text { clay } \underline{(\mathbf{N Z V I / P I L C )}}\end{array}$ & $\begin{array}{l}\text { Nitrate }\left(\mathrm{NO}_{3}^{-}\right) \text {con. }=50 \mathrm{mg} \mathrm{L}^{-1}, \text { initial } \mathrm{pH}= \\
7, \text { material dosage }=0.5 \mathrm{~g} \mathrm{~L}^{-1}, \text { temperature }= \\
25^{\circ} \mathrm{C} \text {, and contact time }=120 \text { min. }\end{array}$ & $100 \%$ & [16] \\
\hline 4 & $\begin{array}{l}\text { nZVI supported on exfoliated } \\
\text { graphite }\end{array}$ & $\begin{array}{l}\text { Nitrate }\left(\mathrm{NO}_{3}^{-}\right) \text {con. }=80 \mathrm{mg} \mathrm{L}^{-1}, \text { initial } \mathrm{pH}= \\
6.7, \text { material dosage }=1.67 \mathrm{~g} \mathrm{~L}^{-1}, \mathrm{Fe}^{0} \text { loading } \\
\text { percentage }=15 \% \text {, temperature }=25^{\circ} \mathrm{C} \text {, and } \\
\text { contact time }=90 \mathrm{~min} .\end{array}$ & $100 \%$ & [17] \\
\hline 5 & $\begin{array}{l}\text { Nanoscale zero-valent iron } \\
\text { (nZVI) with copper deposited } \\
\text { on chelating resin (D407) } \underline{\text { Fe- }} \\
\underline{\text { Cu/D407) }}\end{array}$ & $\begin{array}{l}\text { Nitrate }\left(\mathrm{NO}_{3}^{-}\right) \text {con. }=50 \mathrm{mg} \mathrm{L}^{-1}, \text { initial } \mathrm{pH}= \\
3, \mathrm{Cu}-\mathrm{Fe} \text { ratio }=1: 2, \text { material dosage }=2.5 \mathrm{~g} \\
\mathrm{~L}^{-1} \text {, temperature }=40^{\circ} \mathrm{C}, \text { and contact time }= \\
120 \text { min. }\end{array}$ & $100 \%$ & [18] \\
\hline 6 & $\begin{array}{l}\text { Pyramids structured silicon } \\
(\mathrm{PSi}) \text { with nano zero valent } \\
\text { iron }(\mathrm{NZVI}) \underline{(\mathrm{NZVI} / \mathrm{PSi})}\end{array}$ & $\begin{array}{l}\text { Nitrate }\left(\mathrm{NO}_{3}^{-}\right) \text {con. }=100 \mathrm{mg} \mathrm{L}^{-1} \text {, initial } \mathrm{pH} \\
=3, \text { material dosage }=0.25 \mathrm{~g} \mathrm{~L} \mathrm{~L}^{-1} \text {, } \\
\text { temperature }=\mathrm{N} / \mathrm{A} \text {, and contact time }=140 \\
\text { min. }\end{array}$ & $100 \%$ & [19] \\
\hline 7 & $\begin{array}{l}\text { Multi-wall carbon nanotubes } \\
\text { (MWCNTs) supported nano- } \\
\text { zero valent iron (nZVI) } \\
\text { particles (nZVI@MWCNTs) }\end{array}$ & $\begin{array}{l}\text { Nitrate }\left(\mathrm{NO}_{3}{ }^{-}\right) \text {con. }=50 \mathrm{mg} \mathrm{L}^{-1}, \text { initial } \mathrm{pH}= \\
3 \text {, material dosage }=1 \mathrm{~g} \mathrm{~L}^{-1}, \text { temperature }= \\
25^{\circ} \mathrm{C} \text {, and contact time }=240 \text { min. }\end{array}$ & $100 \%$ & [20] \\
\hline 8 & $\begin{array}{l}\text { nZVI-reduced graphene oxide } \\
\text { composite } \underline{\text { (nZVI-rGO) }}\end{array}$ & $\begin{array}{l}\text { Nitrate }\left(\mathrm{NO}_{3}^{-}\right) \text {con. }=64 \mathrm{mg} \mathrm{L}^{-1}, \text { initial } \mathrm{pH}= \\
7, \text { material dosage }=2.5 \mathrm{~g} \mathrm{~L}^{-1}, \text { and contact } \\
\text { time }=1 \text { min. }\end{array}$ & $96 \%$ & [21] \\
\hline 9 & Coated-Fe ${ }^{0}[\mathrm{Mg} / \mathrm{Fe}]^{1.0}$ & $\begin{array}{l}\text { Nitrate }\left(\mathrm{NO}_{3}{ }^{-}\right) \text {con. }=200 \mathrm{mg} \mathrm{L}^{-1} \text {, initial } \mathrm{pH} \\
=7, \text { material dosage }=1 \mathrm{~g} \mathrm{~L}^{-1} \text {, temperature }= \\
25^{\circ} \mathrm{C} \text {, and contact time }=180 \text { min. }\end{array}$ & $92.4 \%$ & [22] \\
\hline 10 & $\begin{array}{l}\text { Acid mine drainage-based } \\
\text { nZVI (AMD-based nZVI) }\end{array}$ & $\begin{array}{l}\text { Nitrate }\left(\mathrm{NO}_{3}^{-}\right) \text {con. }=50 \mathrm{mg} \mathrm{L}^{-1} \text {, initial } \mathrm{pH}= \\
4.02, \text { material dosage }=3 \mathrm{~g} \mathrm{~L}^{-1} \text {, power of } \\
\text { ultrasonic irradiation }=40 \mathrm{~W} \text {, temperature }= \\
\text { N/A, and contact time }=120 \mathrm{~min} .\end{array}$ & $95 \%$ & {$[23]$} \\
\hline 11 & $\begin{array}{l}\text { Zeolite-supported nanoscale } \\
\text { zero-valent iron/palladium } \\
\underline{\mathbf{Z - F e} / \mathbf{P d})}\end{array}$ & $\begin{array}{l}\text { Nitrate }\left(\mathrm{NO}_{3}^{-}\right) \text {con. }=20 \mathrm{mg} \mathrm{L}^{-1} \text {, initial } \mathrm{pH}= \\
3 \text {, material dosage }=0.5 \mathrm{~g} \mathrm{~L}^{-1}, \text { temperature }= \\
25^{\circ} \mathrm{C} \text {, and contact time }=6 \mathrm{~h} .\end{array}$ & $91.5 \%$ & [24] \\
\hline 12 & $\begin{array}{l}\text { Bimetallic silver/nanoscale } \\
\text { zerovalent iron } \underline{(\mathbf{F e A g})}\end{array}$ & $\begin{array}{l}\text { Nitrate }\left(\mathrm{NO}_{3}{ }^{-}\right) \text {con. }=200 \mathrm{mg} \mathrm{L}^{-1} \text {, initial } \mathrm{pH} \\
=3, \text { material dosage }=0.5 \mathrm{~g} \mathrm{~L}^{-1} \text {, temperature } \\
=25^{\circ} \mathrm{C} \text {, and contact time }=3 \mathrm{~h} .\end{array}$ & $99.5 \%$ & [25] \\
\hline 13 & $\begin{array}{l}\text { Nanoscale zero-valent iron } \\
\text { prepared from steel pickling } \\
\text { waste liquor } \underline{(\mathbf{S}-\mathbf{N Z V I})}\end{array}$ & $\begin{array}{l}\text { Nitrate }\left(\mathrm{NO}_{3}^{-}\right) \text {con. }=20 \mathrm{mg} \mathrm{L}^{-1} \text {, initial } \mathrm{pH}= \\
5 \text {, material dosage }=2.8 \mathrm{~g} \mathrm{~L}^{-1}, \text { temperature }= \\
25^{\circ} \mathrm{C} \text {, and contact time }=4 \mathrm{~h} .\end{array}$ & $99 \%$ & {$[26]$} \\
\hline 14 & $\begin{array}{l}\mathrm{Fe} / \mathrm{Ni} \\
\text { Nanoparticles }\end{array}$ & $\begin{array}{l}\text { Nitrate }\left(\mathrm{NO}_{3}^{-}\right) \text {con. }=300 \mathrm{mg} \mathrm{L}^{-1} \text {, initial } \mathrm{pH} \\
=\text { free, material dosage }=0.2 \mathrm{~g} \mathrm{~L} \mathrm{~L}^{-1}, \\
\text { temperature }=25{ }^{\circ} \mathrm{C} \text {, and contact time }=15 \\
\text { min. }\end{array}$ & $99.8 \%$ & [27] \\
\hline
\end{tabular}


Table 2. Application of nanoscale zerovalent iron particles and its composites in phosphorus removal from water.

\begin{tabular}{|c|c|c|c|c|}
\hline No. & Name of the material or system & Removal conditions & $\begin{array}{l}\text { Removal } \\
\text { Efficiency } \\
\quad(\%)\end{array}$ & Reference \\
\hline 1 & $\begin{array}{l}\text { Nanoscale zero-valent iron (nZVI) } \\
\text { plus copper chloride addition } \\
\left(\mathrm{CuCl}_{2}\right)\end{array}$ & $\begin{array}{l}\text { Phosphorus }(\mathrm{P}) \text { con. }=50 \mathrm{mg} \mathrm{L}^{-1}, \text { initial } \mathrm{pH}= \\
2, \text { material dosage }=1 \mathrm{~g} / \mathrm{L}^{-1}, \mathrm{CuCl}_{2}=25 \mathrm{mg} \text {, } \\
\text { temperature }=25^{\circ} \mathrm{C} \text {, contact time }=60 \mathrm{~min} .\end{array}$ & $100 \%$ & [34] \\
\hline 2 & $\begin{array}{l}\text { Starch-stabilized nanoscale } \\
\text { zerovalent iron }(\mathrm{SnZVI})\end{array}$ & $\begin{array}{l}\text { Phosphate }\left(\mathrm{PO}_{4}^{-3}\right) \text { con. }=100 \mathrm{mg} \mathrm{L}^{-1} \text {, initial } \\
\mathrm{pH}=4 \text {, material dosage }=1 \mathrm{~g} / \mathrm{L}^{-1} \text {, } \\
\text { temperature }=10^{\circ} \mathrm{C} \text {, contact time }=60 \mathrm{~min} \text {. }\end{array}$ & $100 \%$ & {$[35]$} \\
\hline 3 & $\begin{array}{l}\text { Magnetic bimetallic nanoparticles } \\
(\mathrm{Fe}-\mathrm{Ag})\end{array}$ & $\begin{array}{l}\text { Phosphate }\left(\mathrm{PO}_{4}^{-3}\right) \text { con. }=10 \mathrm{mg} \mathrm{L}^{-1} \text {, initial } \mathrm{pH} \\
=\mathrm{N} / \mathrm{A}, \text { material dosage }=1 \mathrm{~g} / \mathrm{L}^{-1} \text {, } \\
\text { temperature }=25^{\circ} \mathrm{C} \text {, contact time }=5 \text { days. }\end{array}$ & $100 \%$ & [36] \\
\hline 4 & $\begin{array}{l}\text { Polyacrylamide coated nanoscale } \\
\text { zero-valent iron particles } \underline{\text { PPA- }} \\
\underline{\mathbf{n Z V I})}\end{array}$ & $\begin{array}{l}\text { Phosphorus }(\mathrm{P}) \text { con. }=100 \mathrm{mg} \mathrm{L}^{-1}, \text { initial } \mathrm{pH} \\
=7, \text { material dosage }=1 \mathrm{~g} \mathrm{~L}^{-1}, \text { temperature }= \\
25^{\circ} \mathrm{C} \text {, and contact time }=120 \mathrm{~min} .\end{array}$ & $100 \%$ & {$[14]$} \\
\hline 5 & $\begin{array}{l}\text { Activated carbon supported } \\
\text { nanoscale } \quad \text { zero-valent } \text { iron } \\
\text { Particles } \underline{(\mathbf{A C}-\mathbf{n Z V I})} .\end{array}$ & $\begin{array}{l}\text { Phosphorus }(\mathrm{P}) \text { con. }=50 \mathrm{mg} \mathrm{L}^{-1} \text {, initial } \mathrm{pH}= \\
\text { 2, material dosage }=8 \mathrm{~g} \mathrm{~L}^{-1} \text {, room } \\
\text { temperature, and contact time }=24 \mathrm{~h} .\end{array}$ & $99.5 \%$ & [37] \\
\hline 6 & $\begin{array}{llr}\text { Magnesium } & \text { hydroxide coated- } \\
\text { nanoscale } & \text { zerovalent iron } \\
\text { nanoparticles }\left(\mathbf{F e}^{\mathbf{0}}[\mathbf{M g} / \mathbf{F e}]^{\mathbf{1 . 0}}\right)\end{array}$ & $\begin{array}{l}\text { Phosphorus }(\mathrm{P}) \text { con. }=50 \mathrm{mg} \mathrm{L}^{-1} \text {, initial } \mathrm{pH}= \\
7, \text { material dosage }=1 \mathrm{~g} \mathrm{~L}^{-1}, \text { temperature }=25 \\
{ }^{\circ} \mathrm{C}, \text { and contact time }=180 \mathrm{~min} .\end{array}$ & $97.4 \%$ & [38] \\
\hline 7 & Nanoscale zerovalent iron $\underline{(\mathbf{n Z V I})}$ & $\begin{array}{l}\text { Phosphorus }(\mathrm{P}) \text { con. }=50 \mathrm{mg} \mathrm{L}^{-1} \text {, initial } \mathrm{pH}= \\
7, \text { material dosage }=1 \mathrm{~g} \mathrm{~L}^{-1} \text {, temperature }=25 \\
{ }^{\circ} \mathrm{C} \text {, and contact time }=180 \mathrm{~min} .\end{array}$ & $94.05 \%$ & {$[31]$} \\
\hline 8 & $\begin{array}{l}\text { Encapsulated nanoscale zero- } \\
\text { valent iron }\end{array}$ & $\begin{array}{l}\text { Phosphate }\left(\mathrm{PO}_{4}^{-3}\right) \text { con. }=10 \mathrm{mg} \mathrm{L}^{-1} \text {, initial } \mathrm{pH} \\
=6.5 \text {, material dosage }=20 \mathrm{~g} \mathrm{~L}^{-1}, \text { temperature } \\
=25^{\circ} \mathrm{C} \text {, contact time }=30 \mathrm{~min} .\end{array}$ & $42 \%$ & [39] \\
\hline 9 & $\begin{array}{l}\text { Nanoscale zero-valent iron/nickel } \\
\text { supported on zeolite } \underline{(\mathbf{Z}-\mathbf{F e} / \mathbf{N i})}\end{array}$ & $\begin{array}{l}\text { Phosphate }\left(\mathrm{PO}_{4}^{-3}\right) \text { con. }=5 \mathrm{mg} \mathrm{L}^{-1} \text {, initial } \mathrm{pH} \\
=3 \text {, material dosage }=0.5 \mathrm{~g} \mathrm{~L}^{-1}, \text { temperature } \\
=25^{\circ} \mathrm{C} \text {, contact time }=6 \mathrm{~h} .\end{array}$ & $98 \%$ & [40] \\
\hline 10 & $\begin{array}{l}\text { Sepiolite-nano zero valent iron } \\
\text { composite }\end{array}$ & $\begin{array}{l}\text { Phosphorus }(\mathrm{P}) \text { con. }=25 \mathrm{mg} \mathrm{L}^{-1}, \text { initial } \mathrm{pH}= \\
4.5, \text { material dosage }=0.8 \mathrm{~g} \mathrm{~L}^{-1}, \text { temperature } \\
=\mathrm{N} / \mathrm{A}, \text { contact time }=46.26 \mathrm{~min} .\end{array}$ & $99.43 \%$ & [41] \\
\hline 11 & $\begin{array}{l}\text { Clay-supported nanoscale zero- } \\
\text { valent iron }\end{array}$ & $\begin{array}{l}\text { Phosphorus }(\mathrm{P}) \text { con. }=500 \mathrm{mg} \mathrm{L}^{-1}, \text { initial } \mathrm{pH} \\
=2, \text { material dosage }=5 \mathrm{~g} \mathrm{~L}^{-1}, \text { temperature }= \\
\text { room temperature, contact time }=24 \mathrm{~h} .\end{array}$ & $30 \%$ & {$[42]$} \\
\hline 12 & $\begin{array}{l}\text { Nanoscale zerovalent iron } \\
\text { particles supported on spondias } \\
\text { purpurea seed waste }(\mathrm{S}-\mathrm{NaOH}- \\
\text { NZVI) }\end{array}$ & $\begin{array}{l}\text { Phosphate }\left(\mathrm{PO}_{4}^{-3}\right) \text { con. }=19 \mathrm{mg} \mathrm{L}^{-1}, \text { initial } \mathrm{pH} \\
=4, \text { material dosage }=1 \mathrm{~g} / \mathrm{L}^{-1}, \text { temperature }= \\
25^{\circ} \mathrm{C} \text {, contact time }=120 \mathrm{~min} .\end{array}$ & $99.9 \%$ & [43] \\
\hline 13 & $\begin{array}{l}\text { Nanoscale zerovalent } \\
\text { particles (nZVI) derived } \\
\text { reducing natural goethite }\end{array}$ & $\begin{array}{l}\text { Phosphorus }(\mathrm{P}) \text { con. }=5.02 \mathrm{mg} \mathrm{L}^{-1} \text {, initial } \mathrm{pH} \\
=6.8, \text { material dosage }=0.6 \mathrm{~g} / \mathrm{L}^{-1} \text {, particle } \\
\text { size }=<75 \mu \mathrm{m} \text {. temperature }=23^{\circ} \mathrm{C} \text {, contact } \\
\text { time }=120 \mathrm{~min} .\end{array}$ & $99.2 \%$ & [44] \\
\hline 14 & Nanoscale zerovalent iron $\underline{(\mathbf{n Z V I})}$ & $\begin{array}{l}\text { Phosphate }\left(\mathrm{PO}_{4}^{-3}\right) \text { con. }=10 \mathrm{mg} \mathrm{L}^{-1} \text {, initial } \mathrm{pH} \\
=\mathrm{N} / \mathrm{A}, \text { material dosage }=1 \mathrm{~g} / \mathrm{L}^{-1}, \\
\text { temperature }=25^{\circ} \mathrm{C} \text {, contact time }=180 \mathrm{~min} .\end{array}$ & $99 \%$ & {$[58]$} \\
\hline
\end{tabular}


Table 3. Application of nanoscale zerovalent iron particles and its composites in heavy metals removal from water.

No. $\quad$ Name of the material or system

$\mathrm{CaCO}_{3}$ coated nanoscale zero-

valent iron (nZVI) $\underline{\text { (C-nZVI) }}$
Removal conditions
Removal

Efficiency Reference

(\%)

Chromium(VI) $[\mathrm{Cr}(\mathrm{VI})]$ con. $=5 \mathrm{mg} \mathrm{L}^{-1}$, initial $\mathrm{pH}=3, \mathrm{Fe}^{0}$ dosage $=250 \mathrm{mg} \mathrm{L}{ }^{-1}$, temperature $=20^{\circ} \mathrm{C}, \mathrm{CaCO}_{3} / \mathrm{Fe}^{0}=1: 4$, and $100 \%$ contact time $=120 \mathrm{~min}$.

\begin{tabular}{|c|c|c|c|c|}
\hline 2 & 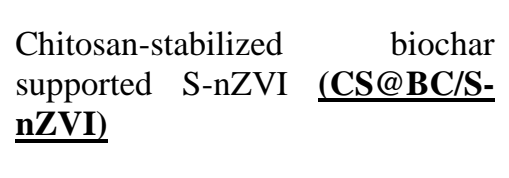 & $\begin{array}{l}\mathrm{Cr}(\mathrm{VI}) \text { con. }=50 \mathrm{mg} \mathrm{L}^{-1} \text {, initial } \mathrm{pH}=3, \\
\text { material dosage }=0.4 \mathrm{~g} \mathrm{~L}^{-1} \text {, temperature }=25 \\
{ }^{\circ} \mathrm{C}, \mathrm{S} / \mathrm{Fe} \text { mole ratio }=0.14, \mathrm{BC} / \mathrm{Fe} \text { mass ratio } \\
=0.25, \text { and contact time }=120 \mathrm{~min} .\end{array}$ & $99.83 \%$ & [50] \\
\hline 3 & $\begin{array}{l}\text { Montmorillonite microparticles } \\
\text { with nanoscale zero-valent iron } \\
\text { (Mt-nZVI) }\end{array}$ & $\begin{array}{l}\mathrm{Cr}(\mathrm{VI}) \text { con. }=20 \mathrm{mg} \mathrm{L}^{-1}, \text { initial } \mathrm{pH}=5.5, \\
\text { material dosage }=2.75 \mathrm{~g} \mathrm{~L}^{-1}, \text { temperature }= \\
25^{\circ} \mathrm{C}, \text { and contact time }=240 \mathrm{~min} .\end{array}$ & $99.95 \%$ & [59] \\
\hline 4 & $\begin{array}{l}\text { Nanoscale Zero Valent Iron } \\
\text { Supported by Biomass-Activated } \\
\text { Carbon } \underline{(\text { BC-nZVI) }}\end{array}$ & $\begin{array}{l}\mathrm{Cr}(\mathrm{VI}) \text { con. }=100 \mathrm{mg} \mathrm{L}^{-1}, \text { initial } \mathrm{pH}=3, \\
\text { material dosage }=400 \mathrm{mg} \mathrm{L}^{-1}, \text { temperature }= \\
25^{\circ} \mathrm{C} \text {, and contact time }=180 \mathrm{~min} .\end{array}$ & $99.12 \%$ & [51] \\
\hline 5 & $\begin{array}{l}\text { Starch coated } \quad \text { nanoscale } \\
\text { zerovalent iron particles supported } \\
\text { on activated carbon } \underline{(\mathbf{S}-} \\
\underline{\text { nZVI@AC) }}\end{array}$ & $\begin{array}{l}\mathrm{Cr}(\mathrm{VI}) \text { con. }=10 \mathrm{mg} \mathrm{L}^{-1} \text {, initial } \mathrm{pH}=5 \text {, mass } \\
\text { ratio of S-nZVI@ } \mathrm{AC} / \mathrm{Cr}=60: 1 \text {, temperature } \\
=25^{\circ} \mathrm{C} \text {, and contact time }=330 \mathrm{~min} .\end{array}$ & $99.96 \%$ & [60] \\
\hline 6 & $\begin{array}{l}\text { Magnetic iron nanoparticles } \\
\text { assisted biochar } \underline{\text { (nZVI-BC) }}\end{array}$ & $\begin{array}{l}\mathrm{Cr}(\mathrm{VI}) \text { con. }=50 \mathrm{mg} \mathrm{L}^{-1}, \text { initial } \mathrm{pH}=4, \\
\text { material dosage }=2 \mathrm{~g} \mathrm{~L}^{-1}, \mathrm{Fe} / \mathrm{BC} \text { ratio }=2: 1, \\
\text { temperature }=20^{\circ} \mathrm{C}, \text { and contact time }=360 \\
\text { min. }\end{array}$ & $99 \%$ & [61] \\
\hline 7 & $\begin{array}{l}\text { Humus-supported nanoscale zero- } \\
\text { valent iron }(\mathrm{H}-\mathrm{nZVI})\end{array}$ & $\begin{array}{l}\mathrm{Cr}(\mathrm{VI}) \text { con. }=25 \mathrm{mg} \mathrm{L}^{-1}, \text { initial } \mathrm{pH}=6.5, \\
\text { material dosage }=6 \mathrm{~g} \mathrm{~L}^{-1}, \mathrm{NZVI} \text { loading }= \\
10.4 \% \text {, temperature }=25^{\circ} \mathrm{C}, \text { and contact time } \\
=90 \mathrm{~min} .\end{array}$ & $99.9 \%$ & [62] \\
\hline 8 & $\begin{array}{l}\text { Biochar derived from nano zero- } \\
\text { valent-iron (nZVI) and sewage } \\
\text { sludge (nZVISB). }\end{array}$ & $\begin{array}{l}\text { Arsenic }(\mathrm{V})[\mathrm{As}(\mathrm{V})] \text { con. }=20 \mathrm{mg} \mathrm{L}^{-1} \text {, initial } \\
\mathrm{pH}=2 \text {, material dosage }=10 \mathrm{~g} \mathrm{~L} \mathrm{~L}^{-1}, \\
\text { temperature }=25^{\circ} \mathrm{C} \text {, contact time }=24 \mathrm{hr} .\end{array}$ & $99 \%$ & [63] \\
\hline 9 & $\begin{array}{l}\text { Zeolite-supported } \\
\text { nanoscale zero-valent iron } \underline{\text { (S- }} \\
\underline{\text { nZVI@ZSM-5) }}\end{array}$ & $\begin{array}{l}\text { As }(\mathrm{V}) \text { con. }=5 \mathrm{mg} \mathrm{L}^{-1} \text {, initial } \mathrm{pH}=4 \text {, material } \\
\text { dosage }=0.1 \mathrm{~g} \mathrm{~L}^{-1}, \text { temperature }=25{ }^{\circ} \mathrm{C}, \\
\text { contact time }=300 \mathrm{~min} .\end{array}$ & $94.5 \%$ & [64] \\
\hline 10 & $\begin{array}{l}\text { Supported nano zero-valent iron } \\
\text { on activated carbon } \underline{(\mathbf{N Z V I} / \mathbf{A C})}\end{array}$ & $\begin{array}{l}\text { As }(\mathrm{V}) \text { con. }=2 \mathrm{mg} \mathrm{L}^{-1}, \text { initial } \mathrm{pH}=6.5 \\
\text { material dosage }=3 \mathrm{~g} \mathrm{~L}^{-1}, \text { temperature }=25 \\
{ }^{\circ} \mathrm{C} \text {, contact time }=72 \mathrm{~h} .\end{array}$ & $100 \%$ & [56] \\
\hline 11 & $\begin{array}{l}\text { Polyaniline } / \mathrm{Fe}^{0} \\
\text { nanofibers } \underline{\left(\mathbf{P A N I} / \mathbf{F e}^{\mathbf{0}}\right)}\end{array}$ & $\begin{array}{l}\text { As }(\mathrm{V}) \text { con. }=5 \mathrm{mg} \mathrm{L}^{-1} \text {, initial } \mathrm{pH}=4 \text {, material } \\
\text { dosage }=0.1 \mathrm{~g} \mathrm{~L}^{-1}, \text { temperature }=25{ }^{\circ} \mathrm{C} \\
\text { contact time }=24 \mathrm{~h} .\end{array}$ & $99.9 \%$ & [65] \\
\hline 12 & $\begin{array}{l}\text { Magnetic } \\
\text { Nanocomposites } \\
\text { (Cellulose@nZVI) }\end{array}$ & $\begin{array}{l}\text { As }(\mathrm{III}) \text { con. }=10 \mathrm{mg} \mathrm{L}^{-1}, \text { initial } \mathrm{pH}=8 \\
\text { material dosage }=1 \mathrm{~g} \mathrm{~L}^{-1}, \text { temperature }=25 \\
{ }^{\circ} \mathrm{C}, \text { contact time }=180 \mathrm{~min} .\end{array}$ & $100 \%$ & [66] \\
\hline 13 & $\begin{array}{l}\text { Chitosan fiber-supported zero- } \\
\text { valent iron nanoparticles }\end{array}$ & $\begin{array}{l}\text { As }(\mathrm{V}) \text { con. }=0.01 \mathrm{mg} \mathrm{L}^{-1}, \text { initial } \mathrm{pH}=6, \\
\text { material dosage }=0.167 \mathrm{~g} \mathrm{~L}^{-1}, \text { temperature }= \\
25^{\circ} \mathrm{C} \text {, contact time }=60 \mathrm{~min} .\end{array}$ & $70 \%$ & [57] \\
\hline 14 & 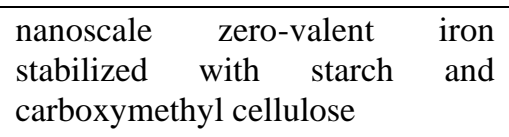 & $\begin{array}{l}\text { Arsenic }(\mathrm{V})[\mathrm{As}(\mathrm{V})] \text { con. }=2 \mathrm{mg} \mathrm{L}{ }^{-1} \text {, initial } \\
\mathrm{pH}=7, \text { material dosage }=0.3 \mathrm{~g} \mathrm{~L} \mathrm{~L}^{-1} \\
\text { temperature }=25^{\circ} \mathrm{C} \text {, contact time }=120 \mathrm{~min}\end{array}$ & $100 \%$ & [55] \\
\hline
\end{tabular}




\section{REFERENCES}

[1] I. Maamoun, O. Falyouna, R. Eljamal, K. Bensaida, O. Eljamal, Optimization modeling of $\mathrm{nFe} 0 / \mathrm{Cu}-\mathrm{PRB}$ design for $\mathrm{Cr}$ (VI) removal from groundwater, Int J Env. Sci Dev. 12 (2021) 131138 .

[2] O. Falyouna, G. Kakonyi, S. Mulligan, A. Fairburn, K. Moharamzadeh, N. Martin, S. Thornton, Behavior of dental composite materials in sterilized and non-sterilized landfill leachate, Sci. 4 (2018) 72-76.

[3] O. Falyouna, O. Eljamal, I. Maamoun, A. Tahara, Y. Sugihara, Magnetic zeolite synthesis for efficient removal of cesium in a lab-scale continuous treatment system, J. Colloid Interface $\begin{array}{lrl}\text { Sci. } & 571 & \text { (2020). }\end{array}$ https://doi.org/10.1016/j.jcis.2020.03.028.

[4] O. Falyouna, I. Maamoun, K. Bensaida, Y. Sugihara, O. Eljamal, Removal of Ciprofloxacin from Aqueous Solutions by Nanoscale Zerovalent Iron-Based Materials: A Mini Review, in: Proc. Int. Exch. Innov. Conf. Eng. Sci., School of Engineering Sciences, Kyushu University, 2020: pp. 179-185. https://doi.org/10.5109/4102485.

[5] I. Maamoun, O. Eljamal, I. Thompson, R. Eljamal, O. Falyouna, Y. Sugihara, Effect of Nano Zero Valent Iron Delivery Method into Porous Media on Phosphorus Removal from Groundwater, in: Proc. Int. Exch. Innov. Conf. Eng. Sci., 2019: pp. 9-11.

[6] I. Maamoun, Innovative Biotechnological Applications of Galdieria Sulphuraria-Red Microalgae (GS-RMA) in Water Treatment Systems, (2020).

[7] O. Falyouna, O. Eljamal, I. Maamoun, Removal of Cesium from Contaminated Waters by Employing Iron-Based Nanoparticles and Nanocomposites, in: 5th Int. Exch. Innov. Conf. Eng. Sci. (IEICES)(5, Pp. 26-27). Interdiscip. Grad. Sch. Eng. Sci. Kyushu Univ. Fukuoka, Japan, 2019.

[8] K. Bensaida, R. Eljamal, kareman Eljamal, Y. Sughihara, O. Eljamal, The impact of iron bimetallic nanoparticles on bulk microbial growth in wastewater, J. Water Process Eng. 40 (2021) 101825 https://doi.org/https://doi.org/10.1016/j.jwpe.20 20.101825 .

[9] T.W.M. Amen, O. Eljamal, A.M.E. Khalil, N. Matsunaga, Wastewater degradation by iron/copper nanoparticles and the microorganism growth rate, J. Environ. Sci. 74 (2018) 19-31. https://doi.org/https://doi.org/10.1016/j.jes.2018 .01 .028 .

[10] K. Bensaida, I. Maamoun, R. ELJAMAL, O. Falyouna, Y. Sugihara, O. Eljamal, Enhancement of Power Generation in Microbial Fuel Cells (Mfcs) Using Iron/Copper Nanoparticles, 6th IEICES-2020, Kyushu Univ.
Fukuoka, Japan. (2020).

[11] A.M. Khalil, Treatment and regeneration of nano-scale zero-valent iron spent in water remediation, (2017).

[12] A.M.E. Khalil, O. Eljamal, S. Jribi, N. Matsunaga, Promoting nitrate reduction kinetics by nanoscale zero valent iron in water via copper salt addition, Chem. Eng. J. 287 (2016) 367-380. https://doi.org/https://doi.org/10.1016/j.cej.2015 .11 .038 .

[13] T. Shubair, O. Eljamal, A.M.E. Khalil, N. Matsunaga, Multilayer system of nanoscale zero valent iron and Nano-Fe/Cu particles for nitrate removal in porous media, Sep. Purif. Technol. 193 (2018) 242-254. https://doi.org/https://doi.org/10.1016/j.seppur.2 017.10.069.

[14] R. Eljamal, O. Eljamal, I. Maamoun, G. Yilmaz, Y. Sugihara, Enhancing the characteristics and reactivity of nZVI: Polymers effect and mechanisms, J. Mol. Liq. 315 (2020) 113714. https://doi.org/https://doi.org/10.1016/j.molliq.2 020.113714 .

[15] N. Song, J. Xu, Y. Cao, F. Xia, J. Zhai, H. Ai, D. Shi, L. Gu, Q. He, Chemical removal and selectivity reduction of nitrate from water by (nano) zero-valent iron/activated carbon microelectrolysis, Chemosphere. 248 (2020) 125986.

[16] Y. Zhang, Y. Li, J. Li, L. Hu, X. Zheng, Enhanced removal of nitrate by a novel composite: Nanoscale zero valent iron supported on pillared clay, Chem. Eng. J. 171 (2011) 526531.

[17] H. ZHANG, Z. JIN, H. Lu, C. QIN, Synthesis of nanoscale zero-valent iron supported on exfoliated graphite for removal of nitrate, Trans. Nonferrous Met. Soc. China. 16 (2006) s345s349.

[18] T.-T. Tang, Q.-J. Xing, S.-H. Zhang, Y. Mu, X.H. Jiang, Z.-G. Zhou, X. Xiao, J.-P. Zou, High selective reduction of nitrate into nitrogen by novel $\mathrm{Fe}-\mathrm{Cu} / \mathrm{D} 407$ composite with excellent stability and activity, Environ. Pollut. 252 (2019) 888-896.

[19] M. Anbia, L. Kamel, Preparation of pyramids structured silicon as a support for nano sized zero valent iron particles for nitrate removal from water, Silicon. 10 (2018) 1851-1859.

[20] A.A. Babaei, A. Azari, R.R. Kalantary, B. Kakavandi, Enhanced removal of nitrate from water using nZVI@ MWCNTs composite: synthesis, kinetics and mechanism of reduction, Water Sci. Technol. 72 (2015) 1988-1999.

[21] S. Khoshro, N.S. Mirbagheri, S. Sabbaghi, Removal of nitrate from aqueous solution using nano zerovalent iron-reduced graphene oxide composite: optimization of parameters, Water Environ. J. 34 (2020) 608-621.

[22] I. Maamoun, O. Eljamal, R. Eljamal, O. 
Falyouna, Y. Sugihara, Promoting aqueous and transport characteristics of highly reactive nanoscale zero valent iron via different layered hydroxide coatings, Appl. Surf. Sci. 506 (2020). https://doi.org/10.1016/j.apsusc.2019.145018.

[23] Z.-H. Diao, W. Qian, Z.-X. Lei, L.-J. Kong, J.-J. Du, H. Liu, J.-W. Yang, S.Y. Pu, Insights on the nitrate reduction and norfloxacin oxidation over a novel nanoscale zero valent iron particle: Reactivity, products, and mechanism, Sci. Total Environ. 660 (2019) 541-549.

[24] Y. He, H. Lin, M. Luo, J. Liu, Y. Dong, B. Li, Highly efficient remediation of groundwater cocontaminated with $\mathrm{Cr}(\mathrm{VI})$ and nitrate by using nano-Fe/Pd bimetal-loaded zeolite: Process product and interaction mechanism, Environ. Pollut. 263 (2020) 114479.

[25] R. Mokete, O. Eljamal, Y. Sugihara, Exploration of the reactivity of nanoscale zero-valent iron (NZVI) associated nanoparticles in diverse experimental conditions, Chem. Eng. Process. Process Intensif. 150 (2020) 107879.

[26] P. Li, K. Lin, Z. Fang, W. Zhang, Degradation of nitrate and secondary pollution in drinking water by S-NZVI prepared from steel pickling waste liquor, J. Hydro-Environment Res. 28 (2020) 1521.

[27] G.G. Valiyeva, I. Bavasso, L. Di Palma, S.R. Hajiyeva, M.A. Ramazanov, F. V Hajiyeva, Synthesis of Fe/Ni bimetallic nanoparticles and application to the catalytic removal of nitrates from water, Nanomaterials. 9 (2019) 1130.

[28] O. Eljamal, A.M.E. Khalil, N. Matsunaga, Experimental and modeling column study of phosphorus removal by permeable reactive materials, Int. J. Environ. Agric. Res. 3 (2017) 62-70.

[29] O. Eljamal, J. Okawauchi, K. Hiramatsu, Product rich in phosphorus produced from phosphoruscontaminated water, in: Adv. Mater. Res., Trans Tech Publ, 2014: pp. 261-265.

[30] S. TAKAMI, O. ELJAMAL, A.M.E. KHALIL, R. ELJAMAL, N. MATSUNAGA, DEVELOPMENT OF CONTINUOUS SYSTEM BASED ON NANOSCALE ZERO VALENT IRON PARTICLES FOR PHOSPHORUS REMOVAL, J. JSCE. 7 (2019) 30-42.

[31] I. Maamoun, R. Eljamal, O. Falyouna, K. Bensaida, Y. Sugihara, O. Eljamal, Insights into kinetics, isotherms and thermodynamics of phosphorus sorption onto nanoscale zero-valent Iron, J. Mol. Liq. 328 (2021) 115402. https://doi.org/https://doi.org/10.1016/j.molliq.2 021.115402 .

[32] E. Osama, O. Junya, H. Kazuaki, Removal of phosphorus from water using marble dust as sorbent material, J. Environ. Prot. (Irvine,. Calif). 2012 (2012).
[33] I. Maamoun, O. Eljamal, T. Shubair, H. Noutsuka, B.B. Saha, N. Matsunaga, Integrating nano-scale zero valent iron (nZVI) in phosphorus removal from aqueous solution through porous media: packed-column experiment, in: Proc. Int. Exch. Innov. Conf. Eng. Sci., Interdisciplinary Graduate School of Engineering Sciences, Kyushu University, 2017: pp. 25-30.

[34] O. Eljamal, A.M.E. Khalil, Y. Sugihara, N. Matsunaga, Phosphorus removal from aqueous solution by nanoscale zero valent iron in the presence of copper chloride, Chem. Eng. J. 293 (2016)

225-231. https://doi.org/https://doi.org/10.1016/j.cej.2016 .02 .052 .

[35] D. Chen, B. Gao, H. Wang, K. Yang, Effective removal of high concentration of phosphate by starch-stabilized nanoscale zerovalent iron (SNZVI), J. Taiwan Inst. Chem. Eng. 61 (2016) 181-187.

[36] Z. Marková, K.M. Šišková, J. Filip, J. Čuda, M. Kolář, K. Šafářová, I. Medřík, R. Zbořil, Air Stable Magnetic Bimetallic $\mathrm{Fe}-\mathrm{Ag}$ Nanoparticles for Advanced Antimicrobial Treatment and Phosphorus Removal, Environ. Sci. Technol. 47 (2013) 5285-5293.

[37] F.A. Bezza, E.M.N. Chirwa, Removal of Phosphate from Contaminated Water Using activated carbon supported Nanoscale ZeroValent Iron (nZVI) Particles, Chem. Eng. 84 (2021).

[38] I. Maamoun, O. Eljamal, O. Falyouna, R. ELJAMAL, Y. Sugihara, Stimulating effect of magnesium hydroxide on aqueous characteristics of iron nanocomposites, Water Sci. Technol. 80 (2020) 1996-2002. https://doi.org/10.2166/wst.2020.027.

[39] A.S. Mahmoud, M.K. Mostafa, M. Nasr, Regression model, artificial intelligence, and cost estimation for phosphate adsorption using encapsulated nanoscale zero-valent iron, Sep. Sci Technol. 54 (2019) 13-26.

[40] Y. He, H. Lin, Y. Dong, B. Li, L. Wang, S. Chu, M. Luo, J. Liu, Zeolite supported $\mathrm{Fe} / \mathrm{Ni}$ bimetallic nanoparticles for simultaneous removal of nitrate and phosphate: Synergistic effect and mechanism, Chem. Eng. J. 347 (2018) 669-681.

[41] M. Malakootian, M. Daneshkhah, H. Hossaini, Removal of phosphates from aqueous solution by sepiolite-nano zero valent iron composite optimization with response surface methodology, Int. J. Environ. Sci. Technol. 15 (2018) 21292140 .

[42] A. Soliemanzadeh, M. Fekri, Synthesis of claysupported nanoscale zero-valent iron using green tea extract for the removal of phosphorus from aqueous solutions, Chinese J. Chem. Eng. 25 (2017) 924-930.

[43] M. Arshadi, S. Foroughifard, J. Etemad Gholtash, 
A. Abbaspourrad, Preparation of iron nanoparticles-loaded Spondias purpurea seed waste as an excellent adsorbent for removal of phosphate from synthetic and natural waters, J. Colloid Interface Sci. 452 (2015) 69-77.

[44] H. Liu, T. Chen, X. Zou, Q. Xie, C. Qing, D. Chen, R.L. Frost, Removal of phosphorus using NZVI derived from reducing natural goethite, Chem. Eng. J. 234 (2013) 80-87.

[45] I. Maamoun, O. Eljamal, O. Falyouna, R. ELJAMAL, Y. Sugihara, Multi-objective optimization of permeable reactive barrier design for $\mathrm{Cr}(\mathrm{VI})$ removal from groundwater, Ecotoxicol. Environ. Saf. 200 (2020). https://doi.org/10.1016/j.ecoenv.2020.110773.

[46] M. Su, Y. Fang, B. Li, W. Yin, J. Gu, H. Liang, P. Li, J. Wu, Enhanced hexavalent chromium removal by activated carbon modified with micro-sized goethite using a facile impregnation method, Sci. Total Environ. 647 (2019) 47-56.

[47] S. Periyasamy, P. Manivasakan, C. Jeyaprabha, S. Meenakshi, N. Viswanathan, Fabrication of nano-graphene oxide assisted hydrotalcite/chitosan biocomposite: An efficient adsorbent for chromium removal from water, Int. J. Biol. Macromol. 132 (2019) 1068-1078.

[48] M. Chigondo, H.K. Paumo, M. Bhaumik, K. Pillay, A. Maity, Magnetic argininefunctionalized polypyrrole with improved and selective chromium(VI) ions removal from water, J. Mol. Liq. 275 (2019) 778-791.

[49] Y. Cheng, H. Dong, T. Hao, CaCO3 coated nanoscale zero-valent iron (nZVI) for the removal of chromium(VI) in aqueous solution, Sep. Purif. Technol. 257 (2021) 117967.

[50] H. Xu, M. Gao, X. Hu, Y. Chen, Y. Li, X. Xu, R. Zhang, X. Yang, C. Tang, X. Hu, A novel preparation of S-nZVI and its high efficient removal of $\mathrm{Cr}(\mathrm{VI})$ in aqueous solution, J. Hazard. Mater. 416 (2021) 125924.

[51] B. Zhang, B.-H. Zhu, X. Wang, S.-B. You, Nanoscale zero valent iron supported by biomass-activated carbon for highly efficient total chromium removal from electroplating wastewater, Water. 12 (2020) 89.

[52] O. Eljamal, K. Sasaki, S. Tsuruyama, T. Hirajima, Kinetic model of arsenic sorption onto zerovalent iron (ZVI), Water Qual. Expo. Heal. 2 (2011) 125-132.

[53] Y.-A. Boussouga, M.B. Mohankumar, A. Gopalakrishnan, A. Welle, A.I. Schäfer, Removal of arsenic(III) via nanofiltration: contribution of organic matter interactions, Water Res. 201 (2021) 117315.

[54] O. Eljamal, K. Sasaki, T. Hirajima, Numerical simulation for reactive solute transport of arsenic in permeable reactive barrier column including zero-valent iron, Appl. Math. Model. 35 (2011) 5198-5207.
[55] M. Mosaferi, S. Nemati, A. Khataee, S. Nasseri, A.A. Hashemi, Removal of Arsenic (III, V) from aqueous solution by nanoscale zero-valent iron stabilized with starch and carboxymethyl cellulose, J. Environ. Heal. Sci. Eng. 12 (2014) $1-11$.

[56] H. Zhu, Y. Jia, X. Wu, H. Wang, Removal of arsenic from water by supported nano zerovalent iron on activated carbon, J. Hazard. Mater. 172 (2009) 1591-1596.

[57] N. Horzum, M.M. Demir, M. Nairat, T. Shahwan, Chitosan fiber-supported zero-valent iron nanoparticles as a novel sorbent for sequestration of inorganic arsenic, RSC Adv. 3 (2013) 78287837.

[58] Z. Wen, Y. Zhang, C. Dai, Removal of phosphate from aqueous solution using nanoscale zerovalent iron (nZVI), Colloids Surfaces A Physicochem. Eng. Asp. 457 (2014) 433-440.

[59] Y. Yin, C. Shen, X. Bi, T. Li, Removal of hexavalent chromium from aqueous solution by fabricating novel heteroaggregates of montmorillonite microparticles with nanoscale zero-valent iron, Sci. Rep. 10 (2020) 1-12.

[60] B. Ji, Y. Shu, Y. Li, J. Wang, Y. Shi, W. Chen, Chromium (VI) removal from water using starch coated nanoscale zerovalent iron particles supported on activated carbon, Chem. Eng. Commun. 206 (2019) 708-715.

[61] S. Zhu, X. Huang, D. Wang, L. Wang, F. Ma, Enhanced hexavalent chromium removal performance and stabilization by magnetic iron nanoparticles assisted biochar in aqueous solution: Mechanisms and application potential, Chemosphere. 207 (2018) 50-59.

[62] R. Fu, X. Zhang, Z. Xu, X. Guo, D. Bi, W. Zhang, Fast and highly efficient removal of chromium (VI) using humus-supported nanoscale zerovalent iron: Influencing factors, kinetics and mechanism, Sep. Purif. Technol. 174 (2017) 362-371.

[63] L. Liu, J. Zhao, X. Liu, S. Bai, H. Lin, D. Wang, Reduction and removal of $\mathrm{As}(\mathrm{V})$ in aqueous solution by biochar derived from nano zerovalent-iron (nZVI) and sewage sludge, Chemosphere. 277 (2021) 130273.

[64] C. Zhou, C. Han, X. Min, T. Yang, Enhancing arsenic removal from acidic wastewater using zeolite-supported sulfide nanoscale zero-valent iron: the role of sulfur and copper, J. Chem. Technol. Biotechnol. (2021).

[65] M. Bhaumik, C. Noubactep, V.K. Gupta, R.I. McCrindle, A. Maity, Polyaniline/Fe0 composite nanofibers: An excellent adsorbent for the removal of arsenic from aqueous solutions, Chem. Eng. J. 271 (2015) 135-146.

[66] S. Zhou, D. Wang, H. Sun, J. Chen, S. Wu, P. Na, Synthesis, Characterization, and Adsorptive Properties of Magnetic Cellulose 
Proceeding of International Exchange and Innovation Conference on Engineering \& Sciences (IEICES) 7 (2021)

Nanocomposites for Arsenic Removal, Water,

Air, Soil Pollut. 225 (2014) 1945. 